\title{
Random sequential adsorption of shrinking or spreading particles
}

\author{
Arsen V. Subashiev, Serge Luryi \\ Department of Electrical and Computer Engineering, \\ State University of New York at Stony Brook, Stony Brook, NY, 11794-2350
}

\begin{abstract}
We present a model of one-dimensional irreversible adsorption in which particles once adsorbed immediately shrink to a smaller size or expand to a larger size. Exact solutions for the fill factor and the particle number variance as a function of the size change are obtained. Results are compared with approximate analytical solutions.
\end{abstract}

PACS numbers: 02.50.Ey, 05.20.-y, 68.43.-h, 07.85.Nc

\section{INTRODUCTION}

Random sequential adsorption (RSA) is an attractive model for a number of physical phenomena, including such different application as information processing [1] and particle branching in impact ionization [2]. The simplest example of RSA is the car parking problem (CPP). Of interest is the average number of particles ("cars") adsorbed on a long line, as well as the variance of this number (see Refs. 3, 4] for the review). In what follows, we shall use the term standard RSA for the classical 1dimensional model corresponding to particles of fixed size that arrive randomly on a line and are deposited if empty space is available and rejected otherwise. Extensions of this model include RSA with particles expanding in the adsorption process [5], two-size particle adsorption [6, 7], and also RSA with an arbitrary particle-size distribution function [8].

Few of these models, especially physically relevant ones, have an exact solution. As a rule, only the fill factor is determined. However, for a number of applications fluctuations are of major importance. An example of such an application is the very important practical problem of particle energy branching (PEB) where high-energy particle propagates in an absorbing medium and multiplies producing secondary electron-hole (e-h) pairs. The energy distribution of secondary particles is random to a good approximation. Multiplication proceeds so long as the particle energy is above the impact ionization threshold [9]. This connection was noticed as early as in 1965 by W. van Roosbroek [10]. The extension of RSA model proposed in 10], known as the "crazy carpenter model" was further exploited in [2, 11].

The PEB process can be considered in terms of a CPP if one identifies the initial particle kinetic energy with an available parking length and the pair creation energy as the car size. Full equivalence to $\mathrm{CPP}$ requires further that only one of the secondary particles takes on significant energy, otherwise one has to consider simultaneous random parking of two cars in one event [12].

The number of created electron-hole pairs $N$ in PEB serves to evaluate the initial energy. Variance of this number limits the accuracy of energy measurements.
Both the yield $\bar{N}$ and the e-h pair variance $\operatorname{var}(N)=$ $\overline{(N-\bar{N})^{2}}$ are proportional to initial energy. The ratio of the e-h pair variance to the yield is called the Fano factor [13]. The Fano factor $\Phi$ (for a Poisson-distributed $N$ one has $\Phi=1$ ) is a parameter that quantifies the energy resolution of high-energy particle detectors. For semiconductor crystals, the PEB problem has many additional complications due to phonon losses, as well as features in energy dependencies of the particle density of states and the impact ionization matrix element.

Earlier attempts to evaluate the Fano factor for the PEB problem in semiconductors employed widely different approaches (compare [9, 10] and [14]). To obtain agreement with the experimentally observed $\Phi \approx 0.1$ (for semiconductors), different fairly rude and unjustified assumptions were made, so that the numerical coincidence is of little value. Also available are numerical calculations 11, 15]. However, the relative importance of various factors (e.g. phonon contribution to $\Phi$ ) remains questionable within the numerical models, while the precision of results is difficult to assess. Evaluation of the Fano factor is important for predicting the energy resolution of detectors, especially those based on new materials and new principles [16.

An important aspect of the impact ionization process is the fact that the threshold impact ionization energy is usually larger than the minimum energy $E_{g}$ needed for e-h pair creation. This difference arises from kinematic restrictions imposed by momentum conservation, so that, e.g., for equal effective masses of electrons and holes the minimal energy of pair production is $3 / 2 E_{g}$. In crystals with non-equal electron and hole masses the threshold can vary from $E_{g}$ to $2 E_{g}$. In terms of the RSA problem this is equivalent to the particle shrinking immediately after the adsorption (parking), making larger available length for the next adsorption event.

For the PEB problem only particle shrinking is pertinent. However, for a general RSA problem both the shrinking and the expansion of particles are relevant, due to such factors as, e.g., repulsion between particles and surface attraction.

In this paper, we use a recursive approach to consider the general RSA problem for particles that either shrink 
or expand immediately upon adsorption. We present an analytical solution for both the average filling factor and its variance. The results are compared with approximate solutions obtained by the methods employed in earlier approaches to the energy branching problem [2, 10, 11, 14] and, for expanding particles, also with the results of a kinetic approach used in Ref. [5]. Exact solutions presented here allow to assess the errors introduced by the adopted approximations in the evaluation of both the yield and the variance.

\section{STATISTICS OF ADSORPTION}

In this section we develop an analytical model of adsorption for shrinking or expanding particles. We shall be using the recursive technique [8]. Besides being the most direct approach, this technique has the further advantage of being applicable for the evaluation of both the average filling factor and the variance. The recursive approach has been confirmed by direct Monte-Carlo computer simulations 8, 10, 15] for similar problems.

Consider adsorption of a particle on an initially empty line of progressively growing length $x$. The final (after shrinking or expansion) size of the adsorbed particle is taken equal unity. Denote by $w_{x}$ the random variable corresponding to the total wasted length for some configuration of adsorbed particles in the jamming limit, when all gaps are smaller than the minimal length needed for a particle to be adsorbed. In the process of sequential adsorption deposition of additional particle generates new gaps with the same distribution of the gap size. Equations for the expected values of the moments of waste distribution can be obtained using moment generation function, $\Psi\left(\lambda, w_{x}\right)=\mathbf{E} \exp \left(\lambda w_{x}\right)$ [17]. Moments of $w_{x}$ are obtained by differentiating $\Psi$ with respect to $\lambda$. When a particle is adsorbed into gap $x$, two new gaps appear, the size of the gaps being $y$ and $x-1-y$. Therefore for $\Psi$ we have

$$
\Psi\left(\lambda, w_{x}\right)=<\Psi\left(\lambda, w_{y}\right) \Psi\left(\lambda, w_{x-1-y}\right)>.
$$

In this equation, the angular brackets denote averaging over the distribution of $y$, characterized by a one-particle gap distribution function (OPDF), $\rho(y \mid x)$, that equals to the probability density of creating an interval $y$ in a one-particle deposition into the line $x$, viz.

$$
<\Psi\left(\lambda, w_{y}\right)>=\int_{y_{\min }}^{y_{\max }} \Psi\left(\lambda, w_{y}\right) \rho(y \mid x) d y .
$$

The randomness in $y$ is induced by a two-step random process, (i) the random choice of the adsorption coordinate and (ii) the subsequent size change, which depends on the adopted model of shrinkage/expansion.

Using Eq. (10) in calculating the first derivative of $\Psi\left(\lambda, w_{x}\right)$ at $\lambda=0$, we find the first moment (the mean value) of waste, $\mathbf{E} w_{x} \equiv w(x)$, in the form

$$
w(x)=\langle w(y)>+<w(x-1-y)>=2<w(y)>,
$$
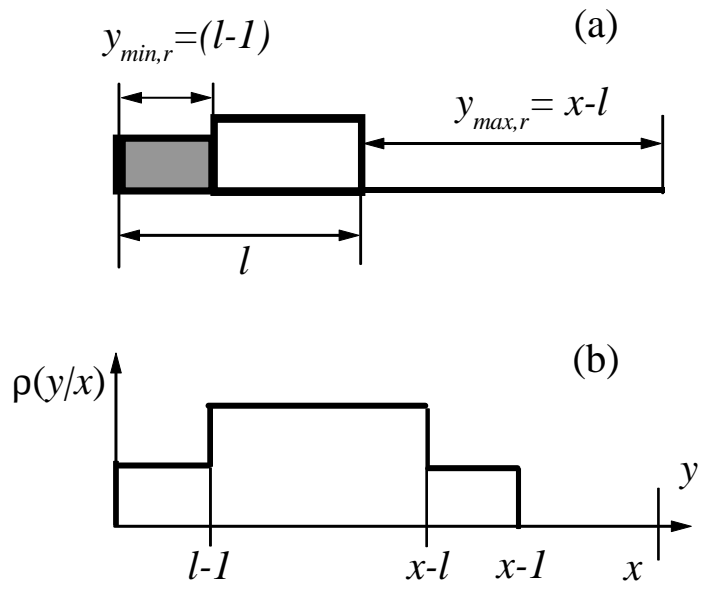

FIG. 1: Illustration (a) of particle adsorption near the edge of a gap of size $x$ in the model where the particle is assumed to shrink upon adsorption (from size $l$ to unit size) by randomly retracting one of its ends (the retracted part is marked by the dark shading, the final state is shown as a white brick of unit length); $y_{\min , r}$ and $y_{\max , r}$ are minimal and maximal gaps created by particles adsorbed at left end of the length $x$ and retracting to right endpoint ; part (b) shows the one particle gap probability distribution function or OPDF, $\rho(y \mid x)$.

the latter equation being due to symmetry. The second moment, $u(x) \equiv \mathbf{E} w_{x}^{2}$, needed for evaluation of variance, is obtained by taking the second derivative,

$$
u(x)=2<u(y)>+2<w(y) w(x-1-y)>.
$$

Equivalent equations can be derived for the mean filled length $f(x)$, which for a unit particle size equals the number of particles adsorbed on the line of length $x$. The random variables $w_{x}$ and $f_{x}$ are complementary in the sense that $w_{x}=x-f_{x}$ and, therefore, equations for $f(x)$ can be obtained from Eqs. (3) and (4) by the substitution $w(x)=x-f(x)$.

For the standard RSA problem the OPDF is a homogeneous distribution of $y$ in the interval $\{0, x-1\})$. The corresponding equations were obtained in 1,10 ] in the context of RSA and in [2, 10] for the energy branching problem.

An alternative approach to RSA is to consider a kinetic (or rate) equation that describes the sequential deposition of particles. In the kinetic approach the gap size distribution function $G(x, t)$ representing the average density of voids of the length between $x$ and $x+d x$ at a time $t$ obeys the equation [18]

$$
\begin{gathered}
\frac{\partial G(x, t)}{\partial t}=-G(x, t) \int_{0}^{x} d s p(s) \int_{0}^{x-s} d y F(y, x-y-s \mid s) \\
+2 \int_{x}^{\infty} d y G(y, t) \int_{0}^{y-x} d s p(s) F(x, y-x-s \mid s) .
\end{gathered}
$$

Here $p(s)$ is the adsorbed particle distribution function, and $F(y, x-y-s \mid s)$ is the deposition probability, that 
determines the average rate,

$$
R(y \mid x)=\int_{0}^{x} d s p(s) F(y, x-y-s \mid s),
$$

at which the initial length $x$ is destroyed by the deposition of a particle producing a gap $y$. One can see that for a fixed $x=x_{0}$ the rate $R$ and our OPDF are proportional to each other, $\rho\left(y \mid x_{0}\right) \propto R\left(y \mid x_{0}\right)$, the only difference being due to the fact that $\rho\left(y \mid x_{0}\right)$ is normalized to unity.

For the case when the randomly adsorbed particles change their size, the OPDF depends on the particular model of size transformation in the adsorbed state. Several such models are discussed below.

Shrinking particles. First, we consider a model in which particles of initial length $l>1$ shrink to the length of unity by randomly retracting one of their endpoints (either on the left or on the right with equal probability).

In this model, no particles are adsorbed for $x<l$, i.e., small intervals are wasted entirely, $w(x)=x$. When the interval length $x$ reaches $l$, then two gaps of size $(l-1) / 2$ are created. For an arbitrary $x>l$, adsorption of a particle creates two new gaps $y$ and $x-1-y$. Note that the probability of creating gaps near the edges of the initial interval (i.e., for $0<y<l-1$ and $x-l<y<x-1$ ) is twice smaller, since they get contributions only from particles that retract the right endpoint (at the right side of the interval $x$ ) and the left endpoint (at the left side of $x$ ), respectively, see Fig. 1. This model can be used for the PEB problem, to account for the effect of lower density of states at low particle energies.

For sufficiently large intervals, $x>2 l-1$, the mean waste is described by the following equation

$$
\begin{gathered}
w(x)=\frac{1}{x-l}\left(\int_{0}^{l-1} w(y) d y+2 \int_{l-1}^{x-l} w(y) d y\right. \\
\left.+\int_{x-l}^{x-1} w(y) d y\right)
\end{gathered}
$$

Note that Eq. (7) does not work in the region $l<x<$ $2 l-1$, where the gap distribution $\rho(y \mid x)$ is simply uniform within $y<x-l$ and $l-1<y<x-1$. However, since the adsorption of the second particle starts at $x>l+1$, knowledge of the variation of $w(x)$ at small $x<l+1$ is sufficient to obtain an exact solution of Eq. (7) - so long as $2 l-1<l+1$ (or $l<2$ ). In what follows we shall confine ourselves to the case $l<2$.

According to (44) the equation for the second moment $u(x)$ is obtained from Eq. (7) by the substitution $w(y) \rightarrow$ $u(y)+w(y) w(x-1-y)$.

Another possible shrinking model results when the adsorbed particle is assumed to shrink symmetrically about its center. In this case the OPDF remains constant within the interval $(l-1) / 2 \leq y \leq x-(l+1) / 2$. This model accounts for the particle repulsion in a model of hard disks, when the distance between the particle centers can not be smaller than a certain length that serves as their effective size. That length can often be larger

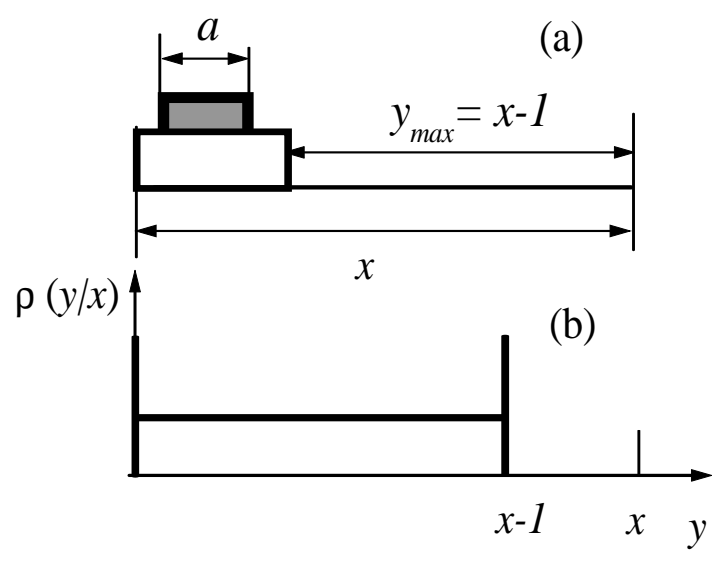

FIG. 2: Adsorption of expanding particles with the transformation rules corresponding to a symmetric expansion $a \Rightarrow 1$ when size permits and near an edge an asymmetric expansion to fill the available space; (a) illustration of particle adsorption near the edge of a gap of size $x$; (b) one-particle gap distribution function, $\rho(y \mid x)$, shows $\delta$-function singularities at $y=x-1$ and $y=0$.

than the actual particle size used in the description of the resulting coverage. A similar model reasonably describes the PEB process in semiconductors, when both secondary particles produced by impact ionization are of the same mass and acquire equal kinetic energies.

With this OPDF, equation (3) takes the form

$$
w(x)=\frac{2}{x-l} \int_{(l-1) / 2}^{x-(l+1) / 2} w(y) d y .
$$

Similarly, equation for the second moment $u(x)$ reads

$$
\begin{aligned}
u(x)=\frac{2}{x-l} & \int_{(l-1) / 2}^{x-(l+1) / 2} u(y) d y \\
& +\frac{2}{x-l} \int_{(l-1) / 2}^{x-(l+1) / 2} w(y) w(x-1-y) d y .
\end{aligned}
$$

These equations are different from those obtained for the standard RSA problem. The modification arises due to the edge effect and is important at the jamming limit, when gaps are minimal. For particles shrinking to the center, the edge effect results in stronger restrictions on the gap size.

Expanding particles. Here we consider a model in which particles of initial length $a<1$ given sufficient space expand symmetrically to unit length. If the particle is placed in a gap of size $a<x<1$, it fills it completely. If it is placed in a gap $x>1$ near its edge, it expands asymmetrically to unit length. This model represents size transformation due to an attractive force from the surface for the case when interaction between the particles is negligible.

To define the ODPF for this model, we note that given the initial length $x$, coordinates of the centers of adsorbed particles are homogeneously distributed within 
$a / 2, x-a / 2$. For particles adsorbed not too close to the gap edges, the gap distribution function remains uniform in the interval $0<y<x-1$. However, all adsorbed particles whose centers fall within an interval $(a / 2,1 / 2)$ from an edge will produce a gap of the same size $x-1$, see Fig. 2, as well as a "gap" of zero width. This results in a singularity at $y=x-1$ (and another one at $y=0$ ) in the gap distribution function (existence of this singularity for expanding particles was already noted in [5]). With this ODPF, Eq. (3) acquires the form

$$
w(x)=\frac{2}{x-a} \int_{0}^{x-1} w(y) d y+\frac{1-a}{x-a} w(x-1) .
$$

Similarly, the second moment equation becomes

$$
\begin{aligned}
u(x)= & \frac{2}{x-a} \int_{0}^{x-1} u(y) d y+\frac{1-a}{x-a} u(x-1) \\
& +\frac{2}{x-a} \int_{0}^{x-1} w(y) w(x-1-y) d y .
\end{aligned}
$$

Note that the particle expansion and edge effect do not influence the second term of Eq. (11), since the product $w(y) w(x-1-y)=0$ vanishes near the edges. Nevertheless, we note that both Eqs. (10) and (11) are modified by the edge effect.

In all models of size transformation considered above the ODPF remains constant within certain intervals. It is this feature that enables exact solution of the recursion equations.

It is worthwhile to stress that due to the self-averaging nature of the filled length (and the waste length) in the limit $x \rightarrow \infty$ solution of the averaged (hence approximate) recursion equations gives exact results. The same is true for the kinetic approach in the limit $t \rightarrow \infty$. Both approaches are, therefore, equivalent for the calculation of the fill factor.

\section{FILLING FACTOR}

We evaluate the filling factor in the so-called "jamming limit" - corresponding to the situation when every gap capable of adsorbing a particle has done so. The average density of particles saturates in the jamming limit.

Shrinking particles. We shall first focus on the model of particles shrinking by randomly retracting one of the endpoints. The case of particles shrinking to the center will be discussed subsequently. We have to solve Eq. (7) with appropriate boundary conditions that originate from the region of small available adsorption interval $x$. Consider an initially empty line of progressively growing length $x$. For $x<l$ the entire interval is wasted, $w(x)=x$. For $l<x<l+1$ only one particle will be adsorbed producing waste equal to $w(x)=x-1$. Clearly, for $x<l+1$ the waste is fixed and does not fluctuate. For $x>l+1$ the probability that two particles will be sequentially adsorbed grows steadily and so does the average waste, which also begins to fluctuate. At large $x$

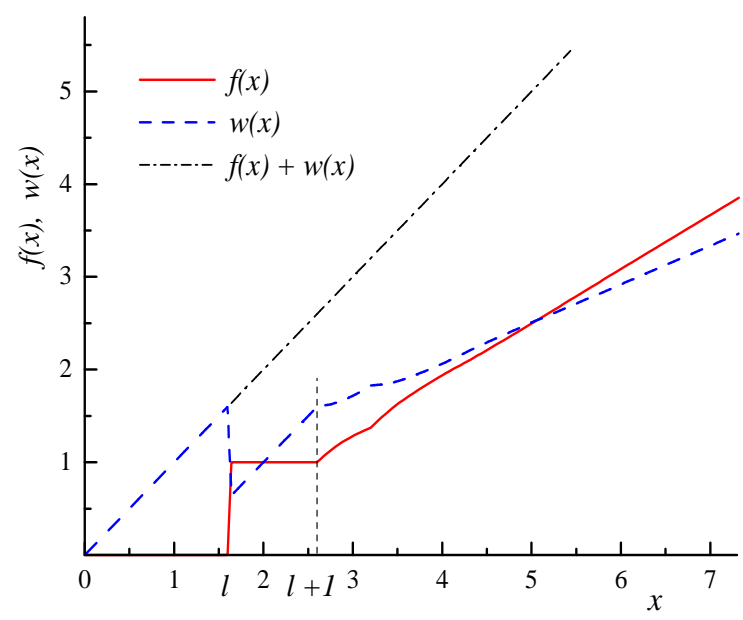

FIG. 3: Average filled length $f$ and the wasted length $w$ for shrinking particles as functions of the length $x$ of the adsorption interval assumed initially empty. The assumed shrinking model is particles randomly retracting their endpoints. The results are obtained by iterating Eq. (9) with the assumed shrinking ratio of $l=1.6$.

both the wasted length and the average covered length grow linearly with $x$, so that the average waste per particle or per unit adsorption interval remains constant.

For particles whose final size is fixed it is natural to study the mean number of particles $f(x)$ adsorbed in a line of length $x$. A convenient equation for $f(x)$ valid for $x>1$ is obtained by substituting $w=x-f(x)$ in Eq. (77) and making the replacement $x \rightarrow x+l$ :

$$
f(x+l)=1+\frac{2}{x} \int_{l}^{\max \{x, l\}} f(y)+\frac{1}{x} \int_{\max \{x, l\}}^{x+l-1} f(y) d y .
$$

In deriving Eq. (12) use has been made of the initial condition

$$
f(x)=\left\{\begin{array}{lc}
0, & 0 \leq x \leq l \\
1, & l \leq x \leq l+1
\end{array}\right.
$$

Evaluation of $f(x)$ at small $x>1+l$ is readily done by a repeated iteration procedure, going from the small to progressively larger length sizes. Results of the numerical recursion are shown in Fig. 3. Note that already at $x \approx 7$ the variations of $f(x)$ and $w(x)$ are very close to linear.

An exact solution of Eq. (12), which will be used to calculate variances, can be obtained using Laplace transformation. Multiplying Eq. (12) by $x$, taking the Laplace transform and using the initial conditions specified by Eq. (13), we obtain an equation of the form

$$
-\frac{d}{d p}\left(e^{p l} F(p)\right)=\frac{1}{p^{2}}+\frac{1}{p} F(p)+\frac{1}{p} e^{p(l-1)} F(p)
$$

for the Laplace transform of $f(x)$,

$$
F(p)=\int_{0}^{\infty} e^{-p x} f(x) d x .
$$


Rearranging terms and multiplying by $e^{-p l}$, Eq. (14) can be rewritten in the form

$$
F^{\prime}(p)+\left[l+\frac{1}{p}\left(e^{-p l}+e^{-p}\right)\right] F(p)=-\frac{\exp (-p l)}{p^{2}} .
$$

The solution of Eq. (16) satisfying the boundary condition at $p \rightarrow \infty$,

$$
\left.F(p)\right|_{p \rightarrow \infty}=\frac{1}{p} e^{-p l},
$$

which follows from the known variation of $f(x)$ at small $x$, can be obtained in a straightforward manner:

$$
F(p)=-\frac{\exp (-p l)}{p^{2} \beta_{r}(p)} \int_{p}^{\infty} \beta_{r}(u) d u
$$

where

$$
\beta_{r}(p)=\exp \left[-\int_{0}^{p}\left(\frac{2-\exp (-v)-\exp (-v l)}{v}\right) d v\right] .
$$

In the analysis of the solution we follow the approach of Ref. [1], based on the use of Karamata's Tauberian theorem, see e.g. 19], p. 37, for the asymptotic growth rate of steadily growing functions. According to that theorem, in order to obtain the asymptotic behavior of the filled length, $f(x)$ (or the gap, $w(x)$, or of the variances of these functions) it is sufficient to have a Laurent powerseries expansion at small $p$ of the Laplace transforms of these functions (possibly cut at small $x$ by a Heaviside step-function factor). Further mathematical details of this type of analysis can be found in [1].

Function $F(p)$ is analytic at all $p \neq 0$ and has a secondorder pole at $p=0$ with the following expansion as $p \rightarrow 0$ :

$$
F(p)=\frac{\alpha_{f, 0}}{p^{2}}+\frac{\alpha_{f, 0}-1}{p}+O(p),
$$

where

$$
\alpha_{f, 0}=\int_{0}^{\infty} \beta_{r}(p) d p .
$$

To calculate $f(x)$ at large $x$, we take the inverse Laplace transformation of the asymptotic expansion (20). This gives

$$
f(x)=\alpha_{f, 0} x+\alpha_{f, 0}-1,
$$

with an exponentially small error term. Whence we have

$$
w(x)=\alpha_{w, 0}(x+1), \quad \alpha_{w, 0}=1-\alpha_{f, 0} .
$$

In the limit $l=1$, equation (21) gives the so-called jamming filling factor $R$ for the standard RSA, $\alpha_{f, 0}(l=1) \equiv$ $R=0.74759 \cdots$ (also called the Renyi constant [20]). The filling factor (saturation coverage), calculated with Eq. (21) as a function of the shrinkage ratio $l$, is shown in Fig. 4 (curve 2). As expected, the shrinking causes a decrease of the filling factor with $l$. Owing to the fact

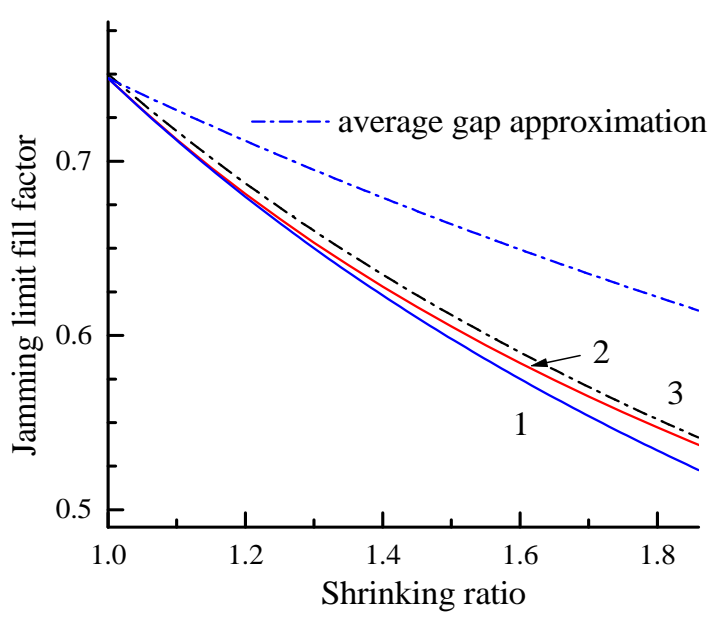

FIG. 4: Filling factor $\alpha_{f, 0}(l)$ as function of the shrinking ratio $l$ for different shrinking models. Curve (1) describes particles shrinking to their centers. For the model of particles randomly retracting their endpoints curve (2) shows the exact solution and curve (3) the linear approximation. The upper curve corresponds to the average-gap approximation.

that the correction terms are exponentially small, our asymptotic solutions (22) and (23) are extremely close (within less than $2 \cdot 10^{-4}$ for $x \geq 7$ ) to the exact solution obtained by direct recursion (Fig. 3). This accounts for the linearity of $f(x)$ and $w(x)$ at high $x$ evident in Fig. 3.

For particles shrinking to their centers, we use Eq. (8) and obtain

$$
f(x)=1+\frac{2}{x-l} \int_{0}^{x-l} f\left(y+\frac{l-1}{2}\right) d y .
$$

Equation (24) can also be written in the form

$$
f\left(x+l+\frac{l+1}{2}\right)=1+\frac{2}{x+(l+1) / 2} \int_{l}^{x+l} f(y) d y .
$$

Equation (25) exhibits a recursion period $(l+1) / 2$ which is to be compared to a recursion period of unity for the standard RSA. The initial condition for Eq. (25) is given by Eq. (13) and the resultant solution is similar in form to Eq. 181) with the following replacement: $\beta_{r}(p) \rightarrow$ $\beta_{c}(p)$, where

$$
\beta_{c}(p)=\exp \left[-2 \int_{0}^{p(l+1) / 2}\left(\frac{1-\exp (-v)}{v}\right) d v\right] .
$$

From Eqs. (26) and (21) it follows that

$$
\alpha_{f, 0}=\frac{2}{1+l} R
$$

Equation (27) represents the exact solution for this model in a simple analytical form. The fill factor calculated with Eq. (27) is shown in Fig. 4 as curve 1. As can 
be expected, the shrinking effect is smaller for particles shrinking by randomly retracting their endpoints than for particles shrinking to their centers. This is due to the reduced contribution of edge regions.

As noted above, the model of particle shrinking to the center accounts for a strong repulsion between the particles acting as hard disks. The minimal distance between the particle centers (an effective particle size) equals to $(l+1) / 2$. Therefore the number of a unit size particles adsorbed in a line with fixed length and the resulting jamming limit fill factor are reduced by $2 /(l+1)$.

An alternative approximate way to obtain an asymptotic solution of Eqs. (12), (25) is to seek the solution in the linear form $f(x)=a x+b$ in the region $x>n l+1$, for some $n$, while using a function obtained by direct iterations for $x<n l+1$. This recipe also gives a very good result starting from $n=1$. Thus, say, for particles shrinking to centers we obtain $\alpha_{f, 0}^{(1)}=2 /(1+l) R^{(1)}$, with $R^{(1)}=0.75$, quite close to the exact Renyi constant. A less accurate approach is to merely match the linear asymptotic to the recursion result for $x<n l+1$. In this case, one would need $n=2$ to obtain a similar precision.

In calculations of the quantum yield and the Fano factor for the PEB problem, the complexity of equations often inspires even more radical approximations, based on estimates of the average losses, see e.g. [9]. In terms of the RSA problem, this is equivalent to assuming the filling factor $\alpha_{f, 0}$ in the form $\alpha_{f, 0}=R\left(R+\alpha_{w, 0}\right)^{-1}$ with the wasted length growing linearly with $l$, i.e. taking $\alpha_{w, 0}=(1-R) \cdot l$. Comparing the exact and the approximate solutions of Eq. (24) (see Fig. 4), we see that this approach severely underestimates the effect of shrinking.

Expanding particles. We consider now the case when the initial particle size is $a<1$. If the interval at the adsorbing line is $a<x<1$, the particle fills it entirely (obviously, without fluctuations). If $x>1$ the particle expands to the length of unity. Evidently, for $x<a$ the whole length is wasted, $w(x)=x$. For $a<x<1$ no waste occurs, while for $1<x<1+a$ the waste equals $w=x-1$ and does not fluctuate. For $x>a+1$ the probability of two particles being adsorbed in this space steadily grows with $x$ and so does the average waste. Thus, the initial conditions to Eq. (10) are of the form

$$
w(x)=\left\{\begin{array}{cc}
x, & 0 \leq x \leq a \\
0, & a \leq x \leq 1 \\
x-1, & 1 \leq x \leq 1+a
\end{array}\right.
$$

Fig. 5 shows the functions $w(x)$ and $f(x)$, obtained by direct iterations of Eq. (10). Note a step-like feature in $f(x)$ and $w(x)$ at $x=1+a$ (which is replicated at $x=n+a$ with ever smaller amplitude) and a decrease of the $w(x)$ in the region $x \geq 1+a$. These are "recursive replicas" of the singularity in OPDF discussed above and the gap in $w(x)$ at $a<x<1$.

To calculate the variance we shall need an exact solution for $w(x)$. It can be obtained by taking Laplace

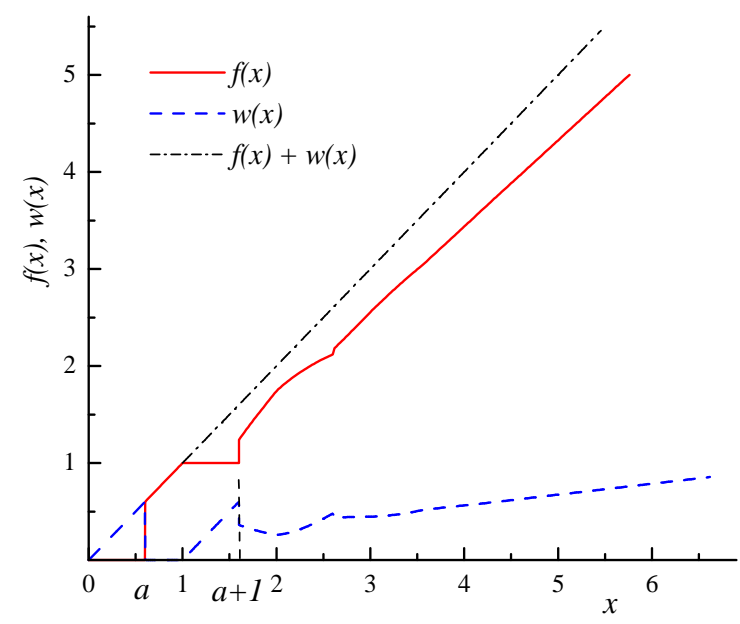

FIG. 5: Average filled length $f$ and the wasted length $w$ for expanding particles as functions of the length $x$ of the adsorption interval assumed initially empty. Results are evaluated by recursion with Eq. (8) for an assumed expansion ratio of $l=1 / 0.6$.

transformation of Eq. (10). First, it is convenient to multiply Eq. (11) by $x-a$ and make the substitution $x \rightarrow x+1$. Taking Laplace transformation, we find that the function

$$
F_{w}(p)=\int_{1}^{\infty} e^{-p x} w(x) d x
$$

(which is the Laplace transform of $w(x)$ cut at small $x<1$ by multiplying with a step function) satisfies the following equation

$$
\begin{aligned}
& {\left[-\frac{d}{d p}+1-a\right]\left(e^{p l} F_{w}(p)\right)=\frac{2}{p} F_{w}(p)} \\
& +(1-a) F_{w}(p)+\left(\frac{2}{p}+1-a\right) J(p),
\end{aligned}
$$

where

$$
J(p)=\int_{0}^{1} w(x) e^{-p x} .
$$

Using Eq. (28) to calculate $J(p)$, we can re-write Eq. (30) in the form

$$
F_{w}^{\prime}(p)+\left(a+\frac{2 e^{-p}}{p} b(a p)\right) F_{w}(p)=-\frac{\exp (-p)}{p^{2}} G_{w}(p),
$$

where

$$
G_{w}(p)=\frac{2}{p} b(a p) J_{1}(a p)
$$

and

$$
b(a p)=1+\frac{1}{2}(1-a) p, \quad J_{1}(a p)=\int_{0}^{a p} t e^{-t} d t .
$$




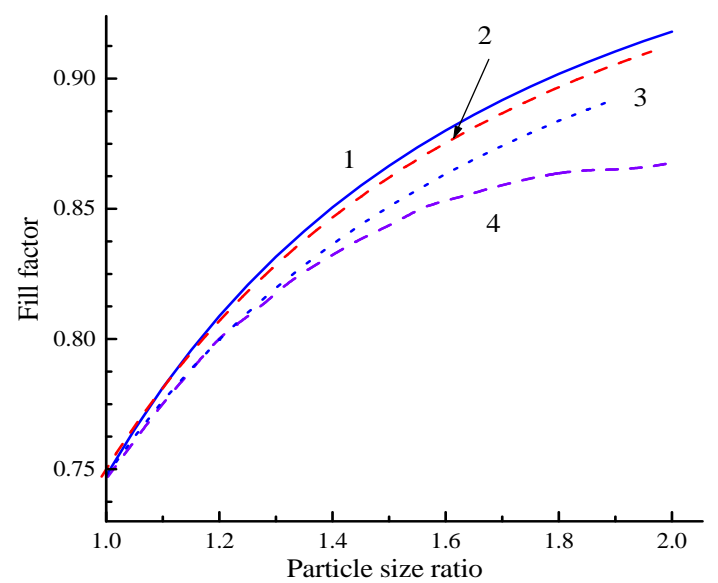

FIG. 6: Filling factor $\alpha_{f, 0}$ as a function of the expansion ratio $l=1 / a$; curve (1) shows the exact result evaluated with Eq. (34), curve (2) the approximate solution of Eq. (8), obtained by linear-to-iterative matching, curve (3) describes the approximate solution neglecting edge effects. Curve (4) corresponds to the restricted expansion model of Ref. [5] (see also Appendix).

Solution of Eq. (32) is of the form

$$
F_{w}(p)=-\frac{\exp (-p)}{p^{2} \tilde{\beta}(p)} \int_{p}^{\infty} \tilde{\beta}(u) G_{w}(u) d u
$$

where

$$
\tilde{\beta}(p)=\exp \left[-2 \int_{0}^{p}\left(\frac{1-e^{-v}}{v}\right) b(a v) d v\right] .
$$

Function $F_{w}(p)$ is analytic at all $p \neq 0$ and has a secondorder pole at $p=0$. As $p \rightarrow 0$, the following asymptotic expansion holds:

$$
F_{w}(p)=\frac{\alpha_{w, 0}}{p^{2}}+\frac{\alpha_{w, 0}}{p}+O(p)
$$

where now

$$
\alpha_{w, 0}=\int_{0}^{\infty} \tilde{\beta}(p) G_{w}(p) d p .
$$

Applying the inverse Laplace transformation to Eq. (37) we bring $w(x)$ at large $x$ into the form $w(x)=\alpha_{w, 0}(x+1)$ with an exponentially small error term. The filling factor $\alpha_{f, 0}=1-\alpha_{w, 0}$ in the jamming limit is then given by

$$
\alpha_{f, 0}=2 \int_{0}^{\infty} \tilde{\beta}(p) b(a p)\left(\frac{e^{-a p}-e^{-p}}{p}+a e^{-a p}\right) d p
$$

For $a=1$, equation (39) again reduces to the Renyi constant $R$.

The filling factor $\alpha_{f, 0}$ calculated with Eq. (39) is plotted in Fig. 6 as function of the expansion ratio $l=1 / a$. We see that expansion causes an increase of the filling factor with $l$. Also shown are the results of Boyer et al. [5] for the RSA problem with restricted particle expansion (their model allows expansion only when the required gap is fully available, see Appendix). As can be expected, the restricted expansion gives a smaller increase of the fill factor.

Figure 6 also shows (curve 3 ) the approximate results obtained by neglecting edge effects; in this approximation the expansion effect is reduced. Much better results are obtained in the approximation (shown by curve 2) obtained by using as a solution of Eq. (10) a linear dependence at $x>n+a$ recursively combined with the exact solution for $x<n+a$. Good agreement with the exact result is obtained already for $n=1$.

\section{VARIANCE AND FANO FACTOR}

Shrinking particles. We shall consider in detail the model of particles shrinking by randomly retracting their endpoints, and then briefly discuss the case of shrinking to the center.

Equation (4) can be readily transformed into an equation for the expected value of the occupied length squared $v=\mathbf{E} f^{2}$. Master equation for $v(x)$ is of the form

$$
\begin{aligned}
v(x)=1 & +\frac{2}{x-l} \int_{l}^{x-l}[v(y)+2 f(x)] d y \\
& +\frac{2}{x-l} \int_{x-l}^{x-1}[v(y)+2 f(x)] d y \\
+ & \frac{2}{x-l} \int_{l}^{x-l} f(y) f(y-x-1) d y .
\end{aligned}
$$

In deriving Eq. (40) we used the initial conditions (13). The Laplace transform $M(p)=\hat{L}(v(x))$ satisfies

$$
M^{\prime}(p)+\left[l+\frac{1}{p}\left(e^{-p l}+e^{-p}\right)\right] M(p)=-\frac{\exp (-p l)}{p^{2}} R_{f}(p),
$$

where

$$
R_{f}(p)=1+2 p F(p)\left(e^{p(l-1)}+1\right)+2 p^{2} F^{2}(p) e^{p(l-1)}
$$

with $F(p)$ defined by Eq. (18). The solution of Eq. (41) can be written in a form similar to Eq. (18), namely

$$
M(p)=-\frac{\exp (-p)}{p^{2} \beta_{r}(p)} \int_{p}^{\infty} \beta_{r}(u) R_{f}(u) d u .
$$

The main feature of this solution is the divergence of the integral in the right-hand side as $p \rightarrow 0$, owing to the square-law dependence of the variance on $x$ at large $x$. This singularity should be treated with care.

To do this we note that $F(p) \propto \alpha_{f, 0} p^{-2}$ at small $p$ and hence the difference $2 p^{2} F^{2}(p)-2 \alpha_{f, 0}^{2} p^{-2}$ is regular at $p \rightarrow 0$. Therefore, it is convenient to define an entire function $\kappa(p)=\beta(p) R_{f}(p)-2 \alpha_{f, 0}^{2} p^{-2}$. In terms of this 
function the solution $M(p)$ can be expressed as follows:

$$
M(p)=-\frac{\exp (-p)}{p^{2} \beta(p)}\left[\frac{2 \alpha_{f, 0}^{2}}{p}+k_{f, 0}-\int_{0}^{p} \kappa(u) d u\right] .
$$

The asymptotic expansion of $M(p)$ near its pole of the third order is of the form

$$
M(p)=\frac{2 \alpha_{f, 0}^{2}}{p^{3}}+\frac{k_{f, 0}+2 \alpha_{f, 0}^{2}}{p^{2}}+\frac{\alpha_{f, 0}^{2}}{p} .
$$

Applying the inverse Laplace transformation, we find the asymptotic form of $v(x)$ :

$$
v(x)=\alpha_{f, 0}^{2} x^{2}+\left(k_{f, 0}+2 \alpha_{f, 0}^{2}\right) x+k_{f, 0}+\alpha_{f, 0}^{2}+1,
$$

with an exponentially small error term. Subtracting $f^{2}(x)$, we find $v(x)-f^{2}(x)=\mu_{r}(x+1)$ where $\mu_{r}=$ $k_{f, 0}+2 \alpha_{f, 0}$ is the specific variance of the fill factor (at $x \rightarrow \infty)$,

$$
\begin{aligned}
\mu_{r}= & 3 \alpha_{f, 0}+2 \int_{0}^{\infty} \beta_{r}(u) p F(u)\left(e^{p(l-1)}+1\right) d u \\
& +2 \int_{0}^{\infty}\left[\beta_{r}(u) u^{2} F^{2}(u) e^{p(l-1)}-\frac{\alpha_{f, 0}^{2}}{u^{2}}\right] d u .
\end{aligned}
$$

Integrating by parts the second term and rearranging the result, we rewrite $\mu_{r}$ in the form

$$
\begin{array}{r}
\mu_{r}=2 \int_{0}^{\infty} \frac{\alpha_{f}(u)}{u}\left(e^{-u}+e^{-u l}-2 e^{-u(l+1)}\right) d u \\
-2 \int_{0}^{\infty} \frac{\alpha_{f}^{2}(u)}{\beta_{r}(u) u^{2}} e^{-u(l+1)}\left((l+1) u+e^{-u}+e^{-u l}-2\right) d u \\
-\alpha_{f, 0},
\end{array}
$$

where

$$
\alpha_{f}(u)=\alpha_{f, 0}-\int_{0}^{u} \beta_{r}(y) d y .
$$

In the limit $l=1$, Eq. (48) reduces to the standard RSA result first obtained for a lattice RSA model by Mackenzie [21]. The numerical value of the Mackenzie constant $\mu_{0}=0.0381564$.. corresponds to the Fano factor $\Phi=0.0510387$.. (see [1] for detailed estimates of the variance). Expression (48) for shrinking particles has the same structure as the corresponding formula for the standard RSA model (fixed-size CPP).

Specific variance $\mu_{r}$ of the filling factor obtained from Eq. (48) is plotted in Fig. 7 against the excess parking length factor $l$. We also plot the ratio of the filling factor variance to its mean value $\mu_{r} / \alpha_{f, 0}$, known in the highenergy detector physics [10, 13] as the Fano factor $\Phi$. We see that while the fill factor variance decreases with $l$ (as does the mean fill factor itself, cf. Fig. 3), their ratio $\Phi$ increases with $l$ due to the steeper decrease of the mean fill factor.

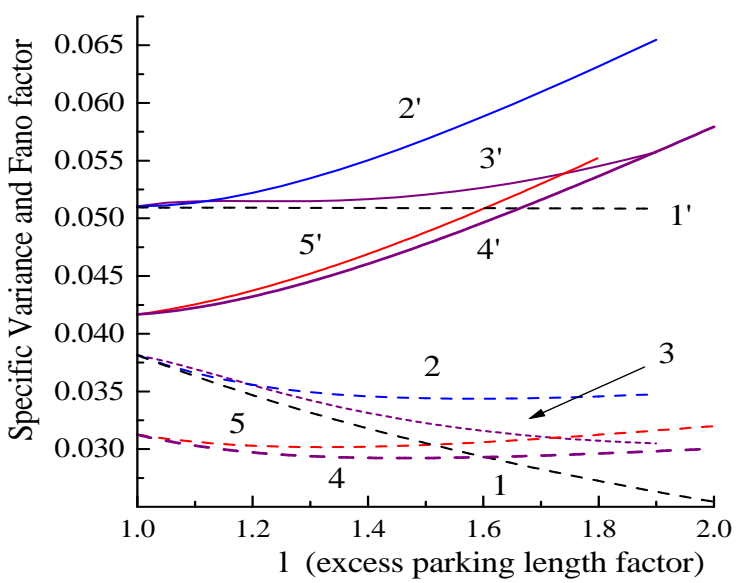

FIG. 7: Specific variance and the Fano factor (shown by curves with primes) as functions of the shrinking ratio $l$. Shown are results of exact calculations: $\left(1,1^{\prime}\right)$ - scaled results for particles shrinking to center, $\left(2,2^{\prime}\right)$ - results for particles randomly shrinking to the ends [based on Eq. (48)], and $\left(3,3^{\prime}\right)$ - results of solution neglecting edge effects; also show results of approximation for $f(x)$ and $u(x)-f(x)^{2}$ at $x>l+1$ allowing edge effect $\left(4,4^{\prime}\right)$ and without it $\left(5,5^{\prime}\right)$.

Similar analysis of the model in which particles shrink to the center shows that for this case the variance asymptotic is given by a constant $\mu_{c}=2 /(l+1) \mu_{0}$, so that it decreases as fast as fill factor. As a result, the Fano factor is not modified in this model. This is in line with the fact that both the number of adsorbed particles and their distribution is identical to that for a standard RSA with the modified particle size.

Figure 7 also shows the variance and the Fano factor obtained by an approximate solution of Eq. (9) based on linear approximations for both the mean waste length $w(x)=a x+b$ and the variance $u(x)-w(x)^{2}=c x+d$ for $x>1+l$ recursively combined with exact expressions in the region $x<1+l$. We see that the difference between the approximate and exact solutions is quite substantial. Moreover, the approximate solution exaggerates the growth of the Fano factor with $l$.

Expanding particles. In this case it is convenient to solve equation (11) for the gap (waste) variance, using Eq. (28) for the boundary conditions. We define a function

$$
N(p)=\int_{1}^{\infty} e^{-p x} u(x) d x
$$

that satisfies an equation of the same type as Eq. (32), in which one should replace $G_{w}(p) \rightarrow R_{w}(p)$, where

$$
R_{w}(p)=\frac{2 J_{2}(a p)}{p^{2}} b(a p)+2 p^{2}\left(F_{w}(p)+\frac{1}{p^{2}} J_{1}(a p)\right)^{2},
$$

with

$$
J_{2}(a p)=\int_{0}^{a p} t^{2} e^{-t} d t .
$$




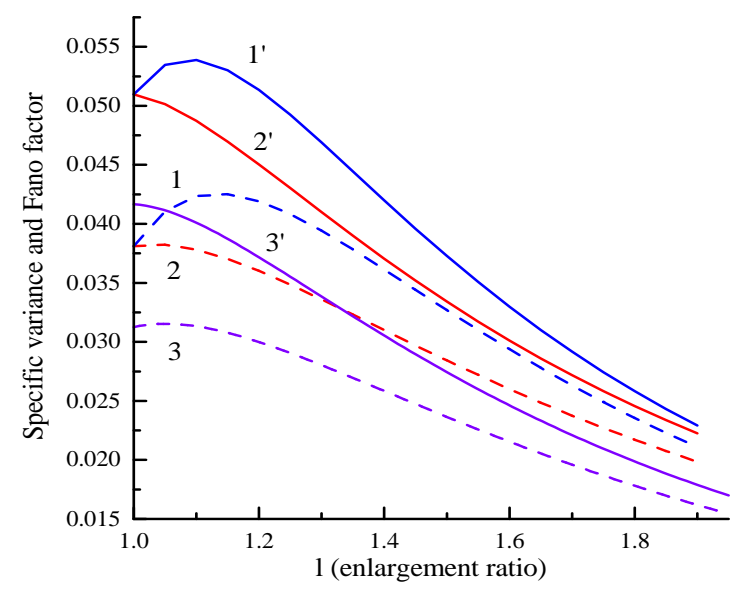

FIG. 8: Specific variance (dashed curves) and the Fano factor (solid curves with primed labels) as functions of the expansion ratio $l=1 / a$. Exact calculations with Eqs. (48-50) are shown by curves $\left(1,1^{\prime}\right)$, curves $\left(2,2^{\prime}\right)$ correspond to edge effect neglected, and curves $\left(3,3^{\prime}\right)$ represent the linear approximation of $w(x)$ and $u(x)-w(x)^{2}$ at $x>l+1$.

Similarly to the standard RSA case, $N(p)$ has a thirdorder pole, i.e. at $p \rightarrow 0$

$$
N(p)=\frac{\alpha_{w, 0}^{2}}{p^{3}}+\frac{\left(k_{w, e}+\alpha_{w, 0}\right)^{2}}{p^{2}}+\frac{k_{w, e}+\alpha_{w, 0}}{p} .
$$

Separating out the regular part of $N(p)$ and rearranging the terms, one obtains the constant $k_{w, e}$ in the form $k_{w, e}=K_{1}+K_{2}+K_{3}$, where

$$
\begin{aligned}
& K_{1}=2 \int_{0}^{\infty} \frac{\tilde{\beta}(u)}{u^{2}}\left(b(a u) J_{1}^{2}(a u)+J_{2}(a u)\right) d u \\
& K_{2}=4 \int_{0}^{\infty} \frac{\alpha_{w}(u)}{u^{2}} e^{-2 u}\left(1-2 e^{-u} b(a u)\right) J_{1}(a u) d u
\end{aligned}
$$

and

$$
K_{3}=-4 \int_{0}^{\infty} \frac{\alpha_{w}(u)^{2}}{\tilde{\beta}(u) u^{2}} e^{-u}\left(\left(e^{-u}-1\right) b(a u)+u\right) d u .
$$

Here

$$
\alpha_{w}(u)=\alpha_{w, 0}-2 \int_{0}^{u} \frac{\tilde{\beta}(p)}{p} b(a p) J_{1}(a p) d p .
$$

Variance of the gap distribution is obtained by the inverse Laplace transformation of $N(p)$. At large $x$ one has $v(x)-w^{2}(x)=k_{w, e}(x+1)$. The constant $k_{w, e}$ describes, with exponentially small errors, the linear dependencies of both the filled length and the gap variance.

With the substitution $\alpha_{w}(u)=(u+1) \tilde{\beta}(u)-\alpha_{f}(u)$ and integration by parts, formulae (54) (56) can be rearranged after some lengthy algebra into a form similar in structure to Eq. (48). However, additional terms at $a \neq 1$ make it rather unwieldy. Both forms are equivalent for numerical integration.

Variance of the gap distribution calculated with Eqs. (54) 566) equals the filled length variance. Figure 8 displays specific variance along with the Fano factor as functions of $l=1 / a$. Note the increase of both the variance and the Fano factor at small $l$. This increase is apparently due to the singular contribution to OPDF from edge effect. For comparison, we show the $v(x)$ and $\Phi$ calculated neglecting the edge effect (this is accomplished by taking $b(a p)=1)$ : no increase is seen in this approximation. Both the variance and the Fano factor vanish with increasing $l$ owing to the fact that variably growing particles provide tighter filling.

Figure 8 also shows the variance and the Fano factor obtained by an approximate solution of Eq. (11) based on the linear approximations for both the mean waste length $w(x)=a x+b$ and the variance $u(x)-w^{2}(x)=c x+d$ for $x>1+l$ combined with exact expressions for $x<1+l$. In contrast to the case of shrinking particles, the difference between the approximate and the exact solutions becomes smaller at larger expansion ratios, since both solutions predict a rapid decrease of the Fano factor with $l$. Note that in this case the overall variation is well described by the approximate solution.

\section{CONCLUSIONS}

We have considered a generalized 1-dimensional random sequential adsorption problem, where particles shrink or expand upon adsorption. Using a recursive approach, we obtained exact analytical expressions for the filling factor and its variance.

In the model where particles shrink to their centers, we find that both the filling factor and its variance scale as the recursion period, so that the Fano factor remains unchanged. But in another model of shrinking, where particles shrink by randomly retracting their endpoints, the Fano factor increases with larger shrinkage ratio, due to the weaker decrease of the variance compared to that of the filling factor. In the case of adsorption of expanding particles (within the allowed intervals), we find that the variance first increases with the expansion ratio $l$ and then decreases, showing a maximum at relatively small values of $l$. These nontrivial results are due to edge effects, resulting in a modified one-particle gap distribution function, $\rho(y)$, near the edges of the adsorbing interval.

The developed approach can be applied to other models, so long as they correspond to a piecewise constant $\rho(y)$. The results are shown to have exponentially small corrections for finite adsorption length $x$; for $x>7 l$ they become practically exact.

The analytic results have been compared to approximate solutions based on a method frequently used in semiconductor physics. The approximate solution is reasonably accurate in estimating the fill factor for either shrinking or expanding particles. However, the same 
method is less accurate in estimates of the fill factor variance. In the model where particles shrink by retracting their endpoints the approximate method overestimates the effects of shrinkage on both the variance and the Fano factor. We also assessed another common approximate method, based on the mean final energy distribution approach, and found that it gives only qualitative trends.

Our quantitative results have important applications to the problem of energy branching in high-energy particle propagation through a semiconductor crystal, where the model of shrinking particles (where particles shrink symmetrically about their centers) naturally accounts for the fact that the impact ionization threshold is larger than the energy gap for electron-hole pair generation. The alternative model of shrinking particles, where shrinking occurs by retracting one of the particle endpoints, further accounts for the decreasing density of states at low particle energies.

Acknowledgement. This work was supported by the New York State Office of Science, Technology and Academic Research (NYSTAR) through the Center for Advanced Sensor Technology (Sensor CAT) at Stony Brook.
[1] E. G. Coffman, Jr., L. Flatto, P. Jelenkovich, and B. Poonen, "Packing random intervals on-line," Algorithmica 22, 448 (1998).

[2] M. Inoue, "Quantum Yield of Electron-Hole Pairs in Semiconductors," Phys. Rev. B 25, 3856 (1982).

[3] J. W. Evans, "Random and cooperative sequential adsorption," Rev. Mod. Phys. 651281 (1993).

[4] J. Talbot, G. Tarjus, P.R Van Tassel, P. Viot, "From car parking to protein adsorption: an overview of sequential adsorption process," Colloids and surfaces A: Physicochemical and Engineering Aspects B 165, 278 (2000).

[5] D. Boyer, J. Talbot, G. Tarjus, P. Van Tassel, and P. Viot, "Exactly solvable models of irreversible adsorption with particle spreading," Phys. Rev. E 49, 5525 (1994).

[6] M. K. Hassan, J. Schmidt, B. Blasius, and J. Kurths, "Jamming coverage in competitive random sequential adsorption of a binary mixture," Phys. Rev. E 65, 045103(R) (2002).

[7] N. A. M. Araujo, and A. Cadilhe, "Gap-size distribution functions of a random sequential adsorption model of segments on a line," Phys. Rev. E 73, 051602 (2006).

[8] D. J. Burridge and Y. Mao, "Recursive approach to random sequential adsorption," Phys. Rev. E 69, 037102 (2004).

[9] H. Spieler, Semiconductor Detector Systems, Oxford University Press, 2005.

[10] W. van Roosbroeck, "Theory of the Yield and Fano Factor of Electron-Hole Pairs Generated in Semiconductors by High-Energy Particles," Phys. Rev. 139, A 1702 (1965).

[11] R. C. Alig, S. Bloom, and C. W. Struck, "Scattering by Ionization and Phonon Emission in Semiconductors," Phys. Rev. B 22, 5565 (1980).

[12] The basic Eqs. (14), (15) of Ref. [10] for the high energy particle energy waste and its variance reduce to the RSA model equations for the gap and its variance for the value of parameter $\gamma=0$, which means that one of the 3 secondary particles has a small kinetic energy.

[13] U. Fano, "Ionization Yield of Radiations. II. The Fluctuations of the Number of Ions," Phys. Rev, 72, 26 (1947).

[14] C. Klein, "Bandgap Dependence and Related Features of Radiation Ionization Energies in Semiconductors," J. Appl. Phys. 39, 2029 (1968).

[15] R.C. Alig, "Scattering by Ionization and Phonon Emission in Semiconductors. 2. Monte-Carlo Calculations," Phys. Rev. B 27, 968 (1983).

[16] A.A. Kastalsky, S. Luryi, B. Spivak, "Semiconductor high-energy radiation scintillator detector," Nucl. Instr. and Meth. A 565, 650 (2006).

[17] J. F. Kenney, and E. S. Keeping, "Moment-Generating and Characteristic Functions," in Mathematics of Statistics, Pt. 2, 2nd ed. Princeton, NJ: Van Nostrand, pp. 72-77, 1951.

[18] M. K. Hassan, and J. Kurths, "Competitive random sequential adsorption of point and fixed-sized particles: analytical results," Journal of Physics A: Mathematical and General, 34, 7517 (2001).

[19] N. H. Bingham, C. M. Goldie, and J. L. Teugels, Regular Variation, Cambridge University Press, Cambridge, 1987.

[20] A. Rényi, "On a one-dimensional problem concerning random space-filling," Publ. Math. Inst. Hung. Acad. Sci. 3109 (1958); Trans. Math. Stat. Prob. 4, 205 (1963).

[21] J. K. Mackenzie, "Sequential Filling of a Line by Intervals Placed at Random and Its Application to Linear Adsorption," Journ. Chem. Phuys. 37, 723 (1962).

\section{APPENDIX A: RESTRICTED EXPANSION MODEL, COMPARISON WITH RATE EQUATION RESULTS}

In the model of RSA with expanding particles studied in [5], the authors considered the case of restricted growth, when the deposited particle begins to grow only if the interval in which it is adsorbed is larger than its final size. For this model, Eq. (11) remains valid for intervals $x>1$, but the initial conditions to Eq. (11) for $w(x)($ at $x<1)$ are of the form

$$
w(x)=\left\{\begin{array}{cc}
x, & 0 \leq x \leq a \\
x-a, & a \leq x \leq 1 \\
x-1, & 1 \leq x \leq 1+a
\end{array}\right.
$$

To solve this model, we shall use the same technique as in Section III] so as to compare the results of the kinetic and the recursive approaches. To do this, we multiply Eq. (11) by $x-a$, make the substitution $x \rightarrow x+1$ and then take Laplace transformation. We obtain an equation similar in form to Eq. (30) where, however, $J(p)$ must be calculated using the revised initial conditions of Eq. 
(A1). This results in

$$
J(p)=\int_{0}^{1} w(x) e^{-p x}=\frac{1}{p^{2}}\left[J_{1}(a p)+e^{-a p} J_{1}((1-a) p)\right] .
$$

The solution of Eq. (30) and hence $\alpha_{w, 0}$ are of the form similar to Eqs. (35) and (38) in which one must use $G_{w}(p)$ defined by Eq. (34) but with $J_{1}(a p)$ replaced by the sum of two terms in the square brackets of Eq. (A2). Calculating the filling factor as $\alpha_{f, 0}=1-\alpha_{w, 0}$, we find

$$
\alpha_{f, 0}=2 \int_{0}^{\infty} \tilde{\beta}(p) b(a p)\left[a e^{-a p}+(1-a) e^{-p}\right] d p .
$$

In Ref. 5], the contribution of expanded and nonexpanded particles was calculated separately for a finite time $t$, using the rate equation approach. In order to make the comparison, one has to take an appropriate linear combination (Eq. (15) and Eqs. (31), (32) of Ref. [5]) in the limit of infinite time. This yields (in the notation of [5]) the fill factor $\theta$ in the form:

$$
\theta=\int_{0}^{\infty} F(t)\left\{1+(\sigma-1)[1+(\sigma-1) t] e^{-(\sigma-1) t}\right\} d t
$$

To compare Eqs. A3 and Eq. A4 , we multiply the right-hand side integrand in (A3) by unity in the form $1-e^{p}+e^{p}$. Then, the contribution $I$, proportional to $1-e^{p}$, can be rewritten as

$$
I=\int_{0}^{\infty} d(\tilde{\beta}(p)) p\left[a e^{(1-a) p}+(1-a)\right]
$$

and then integrated by parts to give

$$
I=-\int_{0}^{\infty} \tilde{\beta}(p)\left\{a[1+(1-a) p] e^{(1-a) p}+1-a\right\} d p .
$$

The remaining part $I I$ is given by

$$
I I=\int_{0}^{\infty} \tilde{\beta}(p)\left[a e^{(1-a) p}+(1-a)\right][2+(1-a) p] d p .
$$

The sum $I+I I$ gives $\alpha_{f, 0}$ in the form

$$
\alpha_{f, 0}=\int_{0}^{\infty} \tilde{\beta}(p)\left[a e^{(1-a) p}+(1-a)(1+(1-a) p] d p .\right.
$$

Equation (A8), after the substitutions $\tilde{\beta}(p)=$ $F(p) \exp [-(1-a) p], a=1 / \sigma$ and $p=\sigma t$, transforms into an expression identical to the $\theta$ given by Eq. A4 derived from [5].

It should be noted, however, that while the fill factors are identical, success of the kinetic approach is hard to extend to variance calculations, where it appears to be much less effective (see e.g. [4]). 